\title{
Horizons/Théâtre
}

Revue d'études théâtrales

8-9 | 2016

Théâtres du geste, du jeu et de la voix

\section{Les adaptations scéniques de contes pour le jeune public, un terrain de jeu pour l'imaginaire}

\section{Jeanne-Lise Pépin}

\section{(2) OpenEdition}

1 Journals

Édition électronique

URL : https://journals.openedition.org/ht/819

DOI : $10.4000 /$ ht.819

ISSN : 2678-5420

Éditeur

Presses universitaires de Bordeaux

\section{Édition imprimée}

Date de publication : 31 décembre 2016

Pagination : 114-127

ISSN : 2261-4591

\section{Référence électronique}

Jeanne-Lise Pépin, «Les adaptations scéniques de contes pour le jeune public, un terrain de jeu pour

l'imaginaire », Horizons/Théâtre [En ligne], 8-9 | 2016, mis en ligne le 01 janvier 2018, consulté le 21 mai 2022. URL : http://journals.openedition.org/ht/819 ; DOI : https://doi.org/10.4000/ht.819

\section{(c) (†) $\odot$}

La revue Horizons/Théâtre est mise à disposition selon les termes de la Licence Creative Commons Attribution - Pas d'Utilisation Commerciale - Pas de Modification 4.0 International. 


\section{JEANNe-LISE PÉPIN}

Jeanne-Lise Pépin a commencé en 2014 un travail de recherche portant sur les adaptations scéniques de contes pour le jeune public dans le cadre d'une thèse de doctorat menée sous la direction de Sandrine Dubouilh (Professeur en études théâtrales au sein de l'Université Bordeaux Montaigne). Cette contribution à la revue Horizons/Théâtre est sa première publication.

Mail : pepin.jeannelise@gmail.com

Résumé : Depuis la parution de l'ouvrage la Psychanalyse des contes de fées de Bruno Bettelheim en 1976, il semble difficile de voir dans le conte autre chose qu'un récit à analyser, décortiquer, et interpréter. En France, dans le domaine du théâtre jeune public, ce dernier occupe une place considérable et ne cesse de donner lieu à de nouvelles adaptations qui s'expriment à la fois sur scène et dans le milieu de l'édition. La tentation est alors grande d'étudier ce phénomène à l'aune des vertus pédagogiques et didactiques que nous accordons communément au conte et qui tendent à faire de lui un outil précieux pour aider l'enfant dans sa construction psychologique et plus précisément pour le préparer à son entrée dans le monde des adultes.

Abstract: Since the publication of The Uses of Enchantment by Bruno Bettelheim in 1976, it seems difficult to perceive tales as something other than a story to analyse, dissect and interpret. In France, in the youth theater domain, tales are widely present and are at the center of new adaptations both for the stage and the publishing world. The temptation is great to study this phenomenon in the light of pedagogic and educational properties which are often attached to tales and which contribute to perceive them as a precious tool to help children in their psychological construction, and more precisely to prepare them for their entrance in the grown-up world.
Cependant une autre voie semble possible, celle d'envisager le conte comme un terrain de jeux pour l'imaginaire. En proposant des réécritures et des adaptations scéniques de conte, des dramaturges et des metteurs en scène ne cessent de jover avec l'horizon d'attente du spectateur/lecteur, ce dernier ayant presque toujours une connaissance partielle ou fragmentaire de I'histoire. Ainsi, un nouvel espace narratif lui est proposé dans lequel se déploient des images ludiques et insolites - images parfois détournées ou réactualisées - et qui finalement contribuent à une forme de réenchantement du conte.

Mots-cLÉs : conte, théâtre jeune public, jeu, imaginaire

However another way seems possible: considering tales as a playground for the imagination. By proposing rewritings and stage adaptations of tales, playwrights and stage directors keep playing with the horizon of expectation of the viewer/reader, who almost always has a partial or fragmentary knowledge of the story. Thus, a new narrative space is offered to him in which ludic and surprising images are unfolded - images which are sometimes twisted and renewed - and which finally contribute to a kind of re-enchantment of tales.

KEYwords: tale, youth theater, play, imagination 


\section{Les adaptations scéniques de contes pour le jeune public, un terrain de jeu pour l'imaginaire}

\section{Introduction}

Difficile, voire impossible d'être vierge de toute expérience liée au conte. Associées au monde de l'enfance, ces histoires vues, lues, entendues, ressassées et inlassablement répétées irriguent encore de manière forte nos existences.

Que ce soit dans le domaine du cinéma, des arts de la scène, de la littérature ou encore de la publicité ${ }^{1}$, le conte occupe une place prépondérante et jouit d'une très forte visibilité qui, pour le moment, ne semble donner aucun signe de fléchissement.

En effet, pas une seule année ne s'écoule sans que l'agenda cinématographique international ne présente un, voire plusieurs films, ayant directement partie liée avec le conte. Ainsi, depuis l'année 2012, il est possible de citer Blanche-Neige de Tarsem Singh (avril 2012²), Blanche-Neige et le Chasseur de Rupert Sanders (juin 2012), Blanca-Nieves de Pablo Berger (janvier 2013), Au bout du conte d'Agnès Jaoui (mars 2013), La Reine des neiges librement inspiré du conte éponyme d'Hans Christian Andersen produit par les studios Disney (décembre 2013), La Belle et la Bête de Christophe Gans sorti en février 2014, Maléfique de Robert Stromberg, à nouveau produit par les studios Disney et s'inspirant librement du conte de La Belle au bois dormant (sorti le 28 mai 2014) et plus récemment encore Into the Woods de Rob Marshall sorti en février 2014 et Cendrillon de Kenneth Branagh sorti en mars 2015.

Dans le domaine du théâtre jeune public, le conte occupe également une place considérable et ne cesse de donner lieu à de nouvelles adaptations qui voient le jour à la fois sur scène et dans le milieu de l'édition. Mais, de la même façon qu'il est difficile, voire impossible d'être totalement vierge de toute expérience liée au conte, il l'est tout autant de voir en lui autre chose qu'un récit à analyser, interpréter et décortiquer, et ce, depuis que la psychanalyse s'en est emparé. 
Dès lors, la tentation est grande d'approcher les adaptations scéniques de contes pour le jeune public à l'aune des vertus pédagogiques et didactiques que nous accordons communément à ce dernier. Tentation d'autant plus grande, que nous avons presque toujours présente à l'esprit l'idée que les enfants auxquels ces spectacles se destinent, sont des adultes en devenir qu'il convient d'orienter dans le droit chemin afin d'en faire de « bons adultes de demain $\gg$, idée et conception que la psychanalyse a largement contribué à véhiculer.

Néanmoins, une autre approche des adaptations scéniques de contes pour le jeune public nous semble possible, celle de les envisager comme une pratique ludique, tant du point de vue de la création que de la réception de l'œuvre.

Afin de donner corps à notre propos, nous rappellerons dans un premier temps les grandes lignes directrices de l'interprétation psychanalytique des contes. Puis, nous nous intéresserons au jeu que les adaptations scéniques de contes permettent de mener avec l'horizon d'attente du spectateur/lecteur. Enfin, dans un troisième et dernier temps, nous évoquerons l'être ensemble et l'aspect rituel qu'offre la mise en partage de ces histoires qui ne nous sont jamais, à quelques exceptions près, totalement inconnues.

\section{L'interprétation psychanalytique des contes}

\section{L'exemple de Cendrillon}

Dans son ouvrage La Psychanalyse des contes de fées publié en France en 1976 - ayant eu un succès retentissant au moment de sa parution et dont l'écho se fait encore vivement sentir de nos jours - Bruno Bettelheim a cherché à démontrer en quoi les contes de fées peuvent être une source de réconfort et d'apaisement pour l'enfant.

Il ne s'agit pas, selon Bettelheim, de lui indiquer ce qu'il est supposé comprendre du conte et quelles informations utiles à son développement psychologique il est susceptible d'y trouver, en pointant du doigt telle ou telle signification supposée cachée derrière tel ou tel symbole, mais de laisser agir en lui la trame narrative du conte. La réception de ces histoires sur la scène de son inconscient aidera alors l'enfant à se débarrasser de ses névroses potentielles et lui permettra de devenir un individu sain qui aura réussi à dompter ses pulsions avant de faire son entrée dans l'âge adulte.

Afin de rendre la pensée de Bettelheim plus intelligible, prenons l'exemple du conte de Cendrillon, sur lequel nous reviendrons ultérieurement, et 
voyons ce qu'il nous en dit. Ce conte, comme il le rappelle, est peut-être «le plus connu, et probablement le plus aimé ${ }^{3} \gg$ de tous les contes et Bruno Bettelheim en propose une analyse qui s'étend sur plus de cinquante pages et dont nous ne donnerons ici que les axes interprétatifs principaux.

D'une part, il y a la thématique de la rivalité fraternelle qui apparaît de manière assez évidente et qui a ici pour particularité d'exister entre le personnage principal et deux jeunes filles avec lesquelles Cendrillon n'a aucun lien de sang, ses demi-sœurs nées de la première union de sa belle-mère. Cette légère mise à distance filiale permettrait à l'enfant lecteur ou auditeur du conte de s'identifier à l'héroïne sans ressentir la culpabilité qu'il pourrait exprimer si les deux affreuses jeunes filles étaient ses véritables sœurs.

Ainsi, il écrit :

Aucun conte de fées ne traduit mieux que «Cendrillon 》, dans toutes ses versions, les expériences vécues par le jeune enfant en proie aux affres de la rivalité fraternelle, quand il se sent désespérément surclassé par ses frères et soeurs. Cendrillon est écrasée et avilie par ses demi-scurs; sa (belle-)mère la sacrifie pour elles; on exige d'elle les corvées les plus sales, et bien qu'elle les accomplisse parfaitement, on ne reconnait pas ses mérites: au contraire on lui en donne davantage. C'est ce que ressent l'enfant quand il est ravagé par les supplices de la rivalité fraternelle ${ }^{4}$.

Les choses seraient bien trop simples si l'interprétation psychanalytique du conte s'arrêtait ici. Toujours selon cette dernière, l'intrigue de Cendrillon permet également d'aborder de manière implicite la question de l'éveil à la sexualité. L'objet qui rend possible une telle interprétation n'est autre que le soulier de vair ou verre selon les différentes versions, quintessence de l'épreuve ultime du conte. Quel pied sera suffisamment fin et menu pour le chausser et permettre ainsi à la jeune fille de convoler avec le beau prince ? Pour Bruno Bettelheim le soulier devient alors le symbole du sexe féminin :

Un petit réceptacle où une partie du corps peut se glisser et être tenue serrée peut être considéré comme le symbole du vagin. Et s'il est fait d'une matière fragile qui peut se briser si on la force, on pense aussitôt à l'hymen; et un objet qui se perd facilement à la fin d'un bal, au moment où l'amant essaie de s'emparer de sa bien aimée, peut passer pour une image assez juste de la virginité particulièrement quand l'homme dresse un piège pour s'en emparer'.

\section{D'un possible contenu didactique dans les adaptations scéniques de contes}

Sans aller aussi loin que Bruno Bettelheim, il semble tout à fait possible de percevoir dans les adaptations scéniques de contes des enjeux pédagogiques 
ou du moins des récits supposés aider l'enfant dans sa construction psychologique. Notre réflexion s'appuie sur quatre pièces qui ont pour particularité d'avoir chacune fait l'objet d'une publication. Il s'agit de deux des réécritures de contes de Joël Pommerat - réécritures et non adaptations comme l'auteur aime à le préciser - Le Petit Chaperon rouge et Cendrillon, de la pièce Gretel et Hansel de Suzanne Lebeau publiée à la rentrée 2014 par les Éditions Théâtrales et de la pièce Dans le ventre du loup de Marion Aubert publiée en 2012 chez Heyoka Jeunesse et qui a pour spécificité d'appeler également un travail chorégraphique, ce texte étant le fruit d'une commande d'écriture de Marion Lévy, chorégraphe et directrice de la compagnie Didascalie.

Il serait ainsi possible de voir dans Le Petit Chaperon rouge de Joël Pommerat, une morale assez basique semblable à celle exposée à la fin du conte de Perrault qui serait de se méfier des apparences parfois trompeuses et notamment des figures de séducteurs, telle celle incarnée par le personnage du loup. L'un des ajouts de Joël Pommerat serait également une forme d'incitation à ne pas délaisser ses proches malgré leur vieillesse qui parfois nous dérange et nous renvoie à notre propre mort. Dans ce texte, la solitude de la grand-mère est particulièrement mise en exergue, et vers la toute fin de la fable, l'homme qui raconte nous dit : «Sa maman qui est vieille habite une maison qui n'est pas très loin, ce qui est plus pratique pour se voir souvent $^{6}$. $\gg$ Marie Bernanoce, spécialiste du théâtre jeune public et auteure de deux répertoires du théâtre contemporain pour la jeunesse ${ }^{7}$, voit, quant à elle, Le Petit Chaperon rouge de Joël Pommerat comme une immersion possible dans le quotidien bien solitaire d'une jeune fille élevée seule par sa mère : « Nous plongeons ainsi dans la solitude d'une petite fille que nous ressentons sans doute comme une victime de la famille monoparentale contemporaine, même si l'absence du père était déjà dans le conte $[\ldots]^{8}$. »

En se rangeant du côté de la psychanalyse, il serait également possible de percevoir Cendrillon, là-encore de Joël Pommerat, comme un texte pouvant presque s'apparenter à un manifeste sur la nécessité de parvenir à faire son deuil, malgré le drame « qui heureusement n'arrive que très rarement aux enfants ${ }^{9} \gg-$ pour reprendre l'expression utilisée dans la pièce - que représente la mort d'un parent au cours de l'enfance. Pour le personnage du jeune prince, comme pour celui de Sandra/Cendrillon, c'est en effet seulement en acceptant de se délester un peu du poids du chagrin et en ne cherchant plus à penser à tout prix et de manière constante à l'être disparu, que la vie peut enfin entrer à nouveau et les submerger par son flot d'expériences nouvelles. 
Gretel et Hansel de Suzanne Lebeau est une pièce qui, en suivant l'interprétation psychanalytique du conte de Cendrillon proposée par Bettelheim, peut être perçue quant à elle comme une démonstration - orientée sur la psychologie et l'intériorité des personnages - cherchant la mise en évidence de l'ambiguité de la relation fraternelle (ici plus précisément de la relation sœur aînée/petit frère) empreinte d'amour certes, mais également souvent teintée de haine.

Enfin, Dans le ventre du loup de Marion Aubert - pièce de théâtre chorégraphique basée sur le conte des Trois Petits Cochons - met en scène trois sœurs et comme il est possible de s'y attendre, l'aînée est la seule à faire l'effort de construire une maison solide, à l'inverse des deux plus jeunes. En ce sens, cette sœur aînée pourrait incarner à elle seule la morale du conte qui s'énoncerait en ces termes : il faut savoir ce que l'on veut, être déterminé et courageux pour être maitre de sa vie et le plus libre possible.

Tout cela fonctionne admirablement bien, mais presque trop bien peutêtre car, comme le souligne avec beaucoup de justesse Pierre Péju, ce que nous gardons en mémoire du conte, c'est rarement l'intégralité de la trame narrative comme la psychanalyse tend à le clamer, mais plutôt des bribes, des fragments, les images les plus frappantes, déroutantes ou alléchantes : «Beaucoup de gens ne se souviennent pas des contes dans leur entier : "J'ai oublié comment ça commençait”, “J’ai perdu la fin...”. Mais il leur reste des images fortes, des épisodes inoubliables, véritables blocs magnétiques autour desquels ils arrangent et brodent ${ }^{10}$. $\gg$ Ce sont précisément ces îlots d'images résiduelles qui vont permettre au dramaturge et au metteur en scène de jouer avec l'horizon d'attente du spectateur/lecteur.

\section{Jouer avec l'horizon d'attente du spectateur/lecteur}

\section{Bref rappel des îlots d'images concernant nos quatre exemples}

Quels pourraient être ces îlots d'images pour les quatre contes qui sont ici au cœur de notre réflexion?

Si l'on tente de se remémorer le conte du Petit Chaperon rouge, ce qui revient surtout à la mémoire, ce serait : la galette et le petit pot de beurre, la visite chez la grand-mère, le bois, la formulette : « Tire la bobinette et la chevillette cherra $\gg$, les phrases rituelles du petit chaperon rouge avec certaines variantes, retenons : «Que tu as de grandes oreilles... », « Que tu as de grandes dents... », etc., la scène de la dévoration et éventuellement la venue du chasseur qui parvient à libérer la grand-mère et sa petite-fille, ce dernier 
n'apparaissant pas dans toutes les versions. Pour Cendrillon, vient dans nos esprits l'image d'un trio de femmes cruelles, une marraine qui par chance est également une fée, la scène de la métamorphose de Cendrillon avant le bal et bien entendu la pantoufle de vair ou verre, selon les versions. Pour Hansel et Gretel, ce serait plutôt : l'abandon, la forêt, la maison en pain d'épices, la méchante sorcière et sa disparition dans la chaleur du four. Enfin, pour Les Trois Petits Cochons, se détachent avant tout de la trame narrative les trois maisons, le plus souvent en paille, en bois et en briques, le loup, son souffle puissant et sa fin funeste dans l'eau bouillante de la marmite.

\section{Jouer avec le spectateur/lecteur pour l'emmener dans des directions inconnues}

Les éléments précédemment énumérés peuvent en quelque sorte s'apparenter à notre substrat mémoriel lié à ces quatre contes. Faisant éminemment partie de nos représentations collectives, ces blocs narratifs et surtout blocs d'images vont permettre aux dramaturges et aux metteurs en scène ayant fait le choix de s'emparer à nouveau de ces récits, de jouer avec nos représentations collectives et avec notre imaginaire.

Les spectateurs/lecteurs se trouvent alors dans un état d'attente complice - attente du bal, du prince, du loup, de la sorcière, etc. - les cartes qui composent le jeu sont connues de tous mais tout l'art du dramaturge/metteur en scène consiste à les rebattre et à proposer une nouvelle combinaison qui, vis-à-vis de l'histoire d'origine, se situe dans un interstice, celui du ni tout à fait la même, ni tout à fait une autre.

Ainsi, dans Le Petit Chaperon rouge de Joël Pommerat, le petit pot de beurre et la galette se transforment en flan un peu trop mou. Ce n'est plus la mère qui demande à sa fille de rendre visite à sa grand-mère mais la petite fille qui en fait la demande et la confrontation entre le loup et la petite fille à la toute fin de l'histoire n'est pas sans nous rappeler une sorte de consultation chez le psy en passe de mal tourner. En effet, pour ne pas laisser la peur l'envahir, la petite fille comble le silence par un flot de paroles quasi ininterrompues concernant sa relation avec sa mère et qui pousse la patience du loup dans ses ultimes retranchements.

LA PETITE FILLE - C'est ma mère qui m'a demandé de faire ce flan pour toi, j'espère que tu vas en manger et que tu vas l'aimer, ma mère ne croyait pas que je serais capable de faire toute seule un flan, elle me croit encore vraiment petite, et finalement je crois qu'elle ne me croit pas encore capable d'avoir des responsabilités dans la vie, les mères c'est toujours comme ça non ? C'est pénible. 


\section{LE LOUP (impatient) - Oui, viens plus près de moi.}

LA PETITE FILLE (de plus en plus effrayée) - Ma mère et moi, on s'entend bien mais des fois c'est vrai j'ai un petit peu du mal à la supporter, elle s'inquiète de tout, alors elle en devient vraiment pénible, elle me prend pour une enfant.

LE LOUP (de plus en plus impatient) - Nous les mamans on s'inquiète beaucoup oui c'est vrai, viens plus près de moi ${ }^{11}$.

Dans Cendrillon de Joël Pommerat, la très jeune fille - comme nous pouvons nous y attendre - est accablée de tâches ménagères mais c'est un état qu'elle cultive d'elle-même et qui lui procure une certaine satisfaction : « Je crois que je vais aimer faire ça les cuves des sept sanitaires, ça va me faire du bien de nettoyer les cuves des sept sanitaires », puis un peu plus loin : «Oui, ça aussi, je crois que je vais aimer ça, retirer les cheveux des lavabos, c'est dégueulasse, ça va me faire du bien ${ }^{12}$. »

De la même manière, il y a bel et bien une fée mais cette dernière est totalement nulle et inefficace, quoique pétrie de bonnes intentions. Elle ne parvient pas à réaliser de simples tours de magie avec des cartes à jouer et la scène de la métamorphose de Sandra/Cendrillon avant le bal se transforme en véritable fiasco. La jeune fille sort une première fois de cette fameuse boîte mystérieuse en majorette, puis à la deuxième tentative de la fée, en mouton. Après ce double échec, la jeune fille décide finalement de mettre une ancienne robe de sa mère, sans avoir recours à une quelconque forme de magie. Sa tenue est très éloignée du faste et des paillettes des robes de princesses auxquels les studios Disney nous ont habitués et pourtant, Sandra ne sera pas tournée en ridicule lors de son arrivée à la première fête organisée par le roi, à la différence des autres membres de sa famille qui se présentent en costumes d'époque. La belle-mère force le père à entrer dans le palais, ce qui suscite l'hilarité générale dans l'assemblée. Là encore, par le biais de cet anachronisme, Joël Pommerat joue avec nos représentations présupposées du conte.

Ce jeu du décalage est particulièrement prégnant dans le traitement du motif de la chaussure. Au sortir de la deuxième soirée organisée par le roi en l'honneur de son fils, c'est la belle-mère qui, humiliée, perd une de ses chaussures que l'un des gardes ramasse. Cette dernière ne sera plus d'aucune utilité pour le reste de l'intrigue, et c'est en revanche le jeune prince qui donne de lui-même sa chaussure à Sandra/Cendrillon en guise de souvenir, c'est précisément cette chaussure qui permettra au roi de retrouver la jeune fille dont son fils ne s'est pas nécessairement épris mais avec laquelle il a, pour la 
première fois depuis le décès de sa mère, créé un lien. Si la pantoufle de verre est évacuée, la matière demeure et se retrouve au centre du dispositif scénographique. C'est en effet la maison qui est en verre, symbole de modernité et source de fierté pour la belle-mère, ce vaste espace transparent - véritable prouesse technique d'un point de vue scénographique - est un objet énigmatique qui résiste à toute tentative d'interprétation univoque. Ces pans de plexiglass sur lesquels les reflets naturels des comédiens se donnent parfois à voir, se transforment d'une scène à l'autre en surface de vidéoprojection et ne cessent de moduler l'espace scénique dans lequel l'histoire se déploie. Réflexion sur le dedans/le dehors, l'intériorité/l'extériorité de chaque être humain, le jeu des apparences, le caractère trompeur de l'image extérieure avec ces oiseaux qui, dupés par les propriétés réfléchissantes du verre, viennent s'écraser sur les vitres et dont Sandra doit, à la demande de sa bellemère, ramasser les cadavres..., les pistes sont multiples mais Joël Pommerat fait tout pour que cette maison de verre reste jusquà la toute fin de la pièce, une image forte, mystérieuse et insaisissable. Ainsi, dans la scène 14, après le départ de Sandra et de son père de la maison, la narratrice raconte :

[... ] il arriva un phénomène curieux. Les oiseaux, comme par un effet magique, ne se cognaient plus contre les parois invisibles de la maison. C'est comme si maintenant ils étaient prévenus du danger. Par contre, de façon curieuse, les bruits que faisait l'impact contre le verre quand ils cognaient, continuèrent, pendant assez longtemps et ça troublait la tranquillité de la petite famille. Heureusement, un jour ça cessa ${ }^{13}$.

Dans Gretel et Hansel, de Suzanne Lebeau, l'une des différences majeures avec la version des frères Grimm, et qui saute aux yeux dès le titre, est l'inversion des rôles des deux personnages. Ce n'est plus Hansel qui réconforte sa sœur et qui astucieusement met en place différents stratagèmes afin d'échapper à l'abandon programmé par les parents, et plus précisément par la mère chez les Grimm, mais Gretel qui rassure, console et épaule son jeune frère à travers les différentes épreuves qu'ils ont à affronter, de l'abandon au cœur de la forêt jusqu'à la confrontation avec la méchante sorcière. Si dans la version des Grimm, c'était déjà Gretel qui avait la brillante idée de pousser la sorcière dans le four, chez Suzanne Lebeau, c'est elle qui assure de bout en bout la protection du petit frère.

En dépit de cette inversion, les personnages de Gretel et d'Hansel fonctionnent dans la pièce comme deux entités inséparables et la dynamique du dialogue repose sur un jeu de complémentarité entre le frère et la sœur. Suzanne Lebeau joue ici avec notre connaissance préalable du conte, ce qui 
est rendu particulièrement tangible dans la scène du choix du prénom du petit frère :

GRETEL - $[\ldots]$ « Comment allez-vous l'appeler ? 》

HANSEL - « Hansel... » a suggéré papa.

GRETEL - « Hansel va très bien avec Gretel,

a dit maman.

Tu ne trouves pas? 》

HANSEL - « Hansel et Gretel me plaît beaucoup », a ajouté papa.

GRETEL - C'est à ce moment-là

que l'histoire d'Hansel et Gretel a commencé.

Pourquoi dit-on toujours : « Hansel et Gretel $\gg$ et pas « Gretel et Hansel $\gg^{14}$ ?

Par ailleurs, alors que c'est d'habitude l'extériorité de la maison en pain d'épices qui nous est présentée, notamment chez les Grimm : $\ll[\ldots]$ et en s'approchant, ils virent que la maisonnette était de pain et couverte d'un toit de gâteau ; quant aux fenêtres elles étaient en sucre candi. "Mettons-nous y, dit Jeannot, et faisons un bon repas. Je vais manger un morceau du toit, tu pourras manger de la fenêtre, Margot, c'est sucré"15. ${ }^{\prime \prime}$

Suzanne Lebeau a quant à elle fait le choix de dépeindre l'intériorité de la maison :

GRETEL - La vieille, très émue, nous regardait... manger avec appétit...

se disant...

qu'elle aussi, bientôt, aurait un bon repas.

[...]

Elle a fait deux lits moelleux de fondant au chocolat...

Oreillers de crème fouettée...

Couvertures de caramel mou...

Nous avons dormi comme des anges ${ }^{16}$.

Avec Dans le ventre du loup, le spectateur/lecteur assiste à un véritable jeu de rôle semblable à ceux auxquels les enfants s'adonnent souvent. La dimension ludique est au cœur même du processus narratif. Le personnage de Zora s'apparente ainsi à un véritable chef de troupe qui répartit les rôles et orchestre l'accomplissement des différentes péripéties composant le récit originel, tout en jouant elle-même le rôle de la mère-truie et du loup. Le texte joue également beaucoup sur les adresses directes aux spectateurs enfants. 


\section{ZORA (en loup) - NOUS SOMMES LÀ POUR VOUS MANGER !!! (elle se calme).}

Tais-toi, Zora, tu dis n'importe quoi. Calme-toi. Je suis toujours excitée, enfants, lorsque je sens de la chair toute fraîche. Et vous êtes de la chair toute fraîche, n'est-ce pas, enfants? (Elle hurle) Pardonnez-moi, enfants. J'ai bu ce soir. C'est parce que je vais interpréter le rôle du loup. Je suis toujours folle lorsque je vais jouer le loup ${ }^{17}$.

Çà et là, la pièce propose également un jeu d'écho avec des éléments ou des catégories de personnages empruntés à d'autres contes. C'est notamment le cas dans la « Séquence 1 » intitulée « La séparation » au cours de laquelle la mère-truie sur le point de mourir dit à ses enfants : « Vous êtes grandes et fortes maintenant. Il est temps pour vous de partir! Regardez. Je vous ai préparé des galettes, et puis un petit pot de beurre. Allez dans la forêt, mes filles! Ouste! Ouste ! Je veux mourir en paix ${ }^{18}$ ! $\gg$, ou encore lors de la «Séquence 7 » intitulée «Destruction de la maison en carton » dans laquelle « la seconde » se plaît à imaginer une sorte de prince charmant lorsque le loup vient frapper à sa porte.

On remarque ainsi que les réécritures de contes permettent de jouer avec l'imaginaire des spectateurs/lecteurs et de déjouer leurs attentes en matière de canevas narratif ou d'images. Par un subtil jeu du décalage et du détour, ces dernières retrouvent une nouvelle vigueur et leur pouvoir d'enchantement s'en voit réactivé.

\section{L'assemblée retrouvée}

\section{Une histoire de transmission}

À l'origine, les contes ne disposaient d'aucun support écrit. Ils se transmettaient de génération en génération par la seule et unique médiation de la voix du conteur qui offrait ses talents à une assemblée réunie pour l'occasion ou au cours de séances improvisées, après un repas ou pendant le travail afin que personne ne vienne à s'endormir. Ce dernier n'existait pas par lui-même et pour lui-même, il était résolument tourné vers l'altérité, celle de l'auditoire qui le réceptionnait et celle du conteur sans lequel il ne pouvait exister car c'est sa voix qui lui donnait vie. Selon l'anthropologue Nicole Belmont, le conte est « ce genre si particulier qui s'élabore dans le processus même de sa transmission ${ }^{19} \gg$. La publication des contes sur support écrit a, par conséquent, constitué un changement majeur dans notre possible rapport au conte. 
Outre les nombreuses critiques que ce changement a pu susciter (contribution à la disparition de la tradition orale, appauvrissement de la matière narrative qui se fige, restriction de l'imaginaire...), ce dernier a avant tout permis, pour toute personne sachant lire, le tête-à-tête avec le conte. Têteà-tête dont les modalités sont fortement éloignées de la rencontre avec le conte vécue à plusieurs, dont la particularité principale n'est autre que son ancrage dans le temps présent. Dans son ouvrage intitulé La Lecture, Vincent Jouve rappelle : « La grande particularité de la lecture par rapport à l'échange oral est son statut de communication différée. L'auteur et le lecteur sont du moins dans la très grande majorité des cas - éloignés l'un de l'autre dans l'espace et dans le temps ${ }^{20}$. $>$ Nicole Belmont, quant à elle, voit dans la lecture solitaire du conte une limite à la relation humaine : « De la lecture, est exclue la complicité qui se nouait entre le conteur, les auditeurs et l'objet qui servait de médiation entre eux, le conte ${ }^{21}$. $\gg$

Cette complicité, dont Nicole Belmont déplore la perte dès lors que la rencontre avec le conte ne s'apparente plus à une aventure collective expérimentée dans un temps présent commun à une assemblée d'auditeurs, semble trouver un nouveau souffle dans le champ des adaptations scéniques de contes pour le jeune public.

\section{La notion d'《être ensemble »}

Choisir de réécrire des contes et en proposer une adaptation scénique pour le jeune public, c'est inviter une assemblée d'enfants spectateurs à s'ancrer dans le hic et nunc de la représentation théâtrale afin de découvrir ou plutôt redécouvrir ensemble une histoire dont ils connaissent le plus souvent les principaux rouages narratifs.

Dans la démarche de création de Joël Pommerat, cette notion d'« être ensemble » est centrale et elle trouve peut-être son achèvement maximal dans ses propositions de réécritures de contes. Ainsi, il écrit :

On pourrait dire aussi que je fais le même travail que les conteurs d'autrefois. François Flahault est [... ] auteur d'un livre intitulé La Pensée des contes. Il rappelle que dans l'acte de conter devant un public, il n'existe pas de séparation, d'opposition et de distinction entre l'«événement d'être ensemble » et la fable. $L a$ définition du conte, c'est cette recherche d'être ensemble.

Un conte, c'est une durée, celle d'un récit, et c'est un état d'être ensemble. Or, le plaisir, c'est d'être relié à quelqu'un au moyen de l'imaginaire, dans un temps donné. Sans ennui, sans effort ${ }^{22}$. 
Bien que ce rapprochement puisse sembler désuet, hasardeux, voire légèrement kitsch, il est ici possible de penser au fameux générique du dessin animé Les Belles Histoires du père Castor ${ }^{23}$ dont la musique s'insinue dans nos esprits avec une efficacité contre laquelle il est difficile de lutter. Cette image de père Castor assis sur son grand fauteuil, entouré de ses trois enfants qui attendent avec impatience et avidité le déploiement d'une nouvelle histoire, a le mérite de condenser à elle seule cette jouissance de l'être ensemble que l'histoire, et ici plus précisément le conte, permet, et qui d'une certaine manière vient réaffirmer la formule lapidaire et extrêmement juste de Walter Benjamin : «Qui écoute une histoire, forme société avec qui la raconte $[\ldots]^{24}$.»

\section{Conclusion}

Nul désir ici d'affirmer que l'interprétation psychanalytique du conte est inopérante, car comme nous l'avons vu dans la première partie de notre développement, cette interprétation fonctionne, mais fonctionne presque trop bien. Par conséquent, nous perdons avec la psychanalyse la jouissance première du conte qui repose sur le plaisir d'un être ensemble très fort, presque de l'ordre du rituel, qui convoque des images fortes, déroutantes et insolites, qui se suffisent à elles-mêmes et que les adultes habitués voire conditionnés à toujours chercher du sens et des explications, ont du mal à concevoir comme une fin en soi, comme un objet ludique à contempler, écouter et répéter inlassablement, sans qu'étonnamment, jamais sa puissance et sa force de rayonnement ne semble s'épuiser.

Le moment où l'on nous explique qu'il y a un sens caché dans le conte, qu'il convient de découvrir et qui nous aidera à devenir de bons adultes de demain, peut être comparé au moment où l'enfant découvre que le père Noël n'existe pas. Il peut y avoir une certaine satisfaction dans cette idée que l'on est résolument entré dans l'ère de la raison et qu'un pas vient d'être franchi en direction du monde des adultes, mais il y a également quelque chose de l'ordre de la perte dans cette révélation, une forme d'enchantement du monde qui tout à coup s'est un peu effritée.

Peut-être est-il alors possible d'envisager les adaptations scéniques de contes pour le jeune public comme un moyen de retrouver un peu de cette magie et de ce puissant imaginaire que le conte ouvrait en nous lorsque, pour la première fois, nous en faisions la rencontre. 
Les adaptations scéniques de contes pour le jeune public, un terrain de jeu pour l'imaginaire

\section{Notes}

1. En effet, plusieurs marques de parfums ont utilisé l'atmosphère du conte comme toiles de fond de leurs campagnes publicitaires.

2. Toutes les dates de sorties mentionnées correspondent aux sorties dans les salles françaises.

3. Bruno Bettelheim, La Psychanalyse des contes de fées, Paris, Robert Laffont, 1976, p. 354.

4. Ibid., p. 355.

5. Ibid., p. 392.

6. Joël Pommerat, Le Petit Chaperon rouge, Arles, Actes Sud, coll. «Babel », 2005, p. 41.

7. Marie Bernanoce, À la découverte de cent et une pièces, répertoire critique du théâtre contemporain pour la jeunesse, et Vers un théâtre contagieux: Volume 2, répertoire critique du théâtre contemporain pour la jeunesse, Paris, Éditions Théâtrales, 2006 et 2012.

8. Marie Bernanoce, Vers un théâtre contagieux, op. cit., p. 416.

9. Joël Pommerat, Cendrillon, op. cit., p. 10.

10. Pierre Péju, La Petite Fille dans la forêt des contes, Paris, Robert Laffont, coll. « Réponses », 1981, p. 60.

11. Joël Pommerat, Le Petit Chaperon rouge, op. cit., p. 33-34.

12. Joël Pommerat, Cendrillon, op. cit., p. 37.

13. Ibid., p. 110-111.

14. Suzanne Lebeau, Gretel et Hansel, Paris, Éditions Théâtrales, coll. « Jeunesse », 2014, p. $15-16$.

15. Jacob et Wilhelm Grimm, Contes, Paris, Gallimard, coll. « folio classique », 1976, p. 77.

16. Suzanne Lebeau, op. cit., p. 55.

17. Marion Aubert, Dans le ventre du loup, Arles, Actes Sud, coll. " Heyoka-Jeunesse ", 2012, p. 7.

18. Ibid., p. 8.

19. Nicole Belmont, Poétique du conte, essai sur le conte de tradition orale, Paris, Gallimard, coll. «Le Langage des contes », 1999, p. 9.

20. Vincent Jouve, La Lecture, Paris, Hachette Supérieur, coll. « Contours littéraires », 1993, p. 13.

21. Nicole Belmont, op. cit., p. 79.

22. Joëlle Gayot et Joël Pommerat, Joël Pommerat, troubles, Arles, Actes Sud, 2009, p. 60.

23. Réalisé par Jean Cubaud, Philippe Moreau et Greg Bailey, le dessin animé Les Belles Histoires du père Castor a été diffusé à partir de 1993 sur différentes chaînes de la télévision française.

24. Walter Benjamin, Essais II - 1935-1940. In Isabelle Nières-Chevrel, Introduction à la littérature de jeunesse, Paris, Didier Jeunesse, coll. « Passeurs d'histoires », 2009, p. 75. 